\title{
High Pulse Repetition Frequency Micro Hole Drilling of Silicon Using Ultrashort Pulse Laser Radiation
}

\author{
Andreas Gruner, Lucas Naumann, Joerg Schille and Udo Loeschner \\ Laserinsitut Hochschule Mittweida, University of Applied Sciences Mittweida \\ Technikumplatz 17, 09648 Mittweida, Germany \\ E-mail:gruner@hs-mittweida.de
}

\begin{abstract}
Laser processing of silicon, as the most important material for microelectromechanical systems (MEMS), is commonly known and accepted as an effective manufacturing method to fabricate complex and precise micro components. Especially for the fabrication of micro hole grids, the recently developed high pulse repetition frequency (PRF) laser micro drilling technology is a valuable alternative to standard processing techniques. This paper discusses laser percussion drilling of silicon using ultrashort laser pulses at high average powers with the aim to drill through a material thickness of $100 \mu \mathrm{m}$. The main process influencing parameters, such as PRF and laser fluence were varied in order to identify optimal parameter settings for high quality and high throughput micro hole drilling. Furthermore, the effect of additional laser pulses, irradiated after drilling through, onto the micro hole geometry was examined. It will be shown that with increasing PRF and thus, lower laser fluence as a result of constant average laser power of the used laser system, the drilling time decreases significantly. This might be due to thermal accumulation induced by the applied highlyrepetitive pulses, leading to a higher substrate temperature which, in turn, lowers the energy needed for melting and evaporation. Surprisingly, this effect results in higher quality of the micro hole geometry without considerable debris at both, hole entrance and exit.
\end{abstract}

DOI: $10.2961 / \mathrm{jlmn} .2019 .03 .0001$

Keywords: ultrashort pulse laser, micro hole drilling, high pulse repetition frequency (PRF), micro processing, silicon

\section{Introduction}

In recent years, there is growing demand in microelectromechanical systems (MEMS) development and fabrication. For MEMS, silicon is the most widespread and important material beside polymers, specific metals and ceramics. As laser processing of silicon has been widely investigated during the past, it could be shown, that ultrashort pulse laser radiation is well suitable to fabricate complex and precise microstructures $[1,2]$, micro drilling holes [36] or rather interconnection vias [7-9]. This resulted from the outstanding properties of the ultrashort pulses regarding their extremely short laser-material-interaction times and therefore low thermal impact to the sample material.

Special interest should be directed towards MEMS gas field ionization sources (GFIS) used for micro ion thrusters as a promising technology for micro propulsion systems. MEMS ion thruster concept combines the simplicity of field emission thruster operation with ion thruster technology [10]. The operating principle of GFIS thrusters is based on gas (Xe or Ar) flowing through a porous silicon structure with carbon nanotubes (CNTs) deposited on the exit side surface functioning as field ionizers anode. The ions, in turn, are accelerated in an electric field towards the cathode. Initial approaches in development of MEMS GFIS were investigated at the NASA Ames research centre and MIT [11-13]. They demonstrated the proof of concept for MEMS GFIS but struggled in manufacturing details of the porous silicon-chip, processed by deep reactive-ion etching (DRIE). Further development has been done at TU Dresden where the silicon grid was produced by UV-laser radiation of nanosecond pulse duration and low pulse repetition frequency (PRF) using the percussion drilling mode [10, 1417]. The focus was on high perforation share and an unaffected and debris free hole exit side surface as a condition for CNT-coating applying the atmospheric pressure chemical vapour disposition method (APCVD). However, the chip-processing was challenging due to high thermal load to the substrate leading to melting residues at the hole entrance and restricted mechanical stability. In addition, the long processing time for drilling the micro hole grids was a significant technological disadvantage. Therefore, high PRF ultrashort pulse laser technology offers new approaches in manufacturing of micro hole grids for GFIS. The commercial availability of high power, high PRF and brilliant ultrashort pulse lasers combines industrial needs of high machining throughput with high processing quality, which seems to be interesting in the fields of automotive, aerospace, electro-optics, photonics, biomedical, semiconductor, etc.

Previous studies investigating micro hole drilling of stainless steel applying PRFs up to $1 \mathrm{MHz}$ identified the ablation efficiency can be increased compared to lower PRFs. This was due to the fact, that heat accumulation counterbalances plasma- and particle shielding losses as observed for PRF up to $500 \mathrm{kHz}$ [18-20]. This was at the expense of drilling quality as a consequence of heat accumulation leading to a high thermal impact and thus local melting. However, a recent study demonstrated, that con- 
ventional laser percussion drilling of metal foils can be transferred into a high PRF process using ultrashort pulse laser radiation and PRFs up to $48.7 \mathrm{MHz}$ [21]. Especially for the fabrication of micro hole grids with high aspect ratio and a hole number of more than 10,000 high PRF laser micro drilling is an alternative to the standard processing techniques, such as DRIE and other conventional etching methods.

In this study, the main process influencing parameters, such as PRF and laser fluence, were varied to identify optimal parameter settings for a high quality micro hole geometry in combination with high throughput. Also the effect of additional laser pulses onto the micro hole geometry, irradiated after drilling through, was examined. Finally, a machining example was processed with optimum process parameters to demonstrate the potential of the technology.

\section{Experimental Setup}

A femtosecond laser system based on InnoSlab technology was used in this work. The laser source delivered a maximum output power of about $92 \mathrm{~W}$ onto the work piece surface at a PRF of $48.72 \mathrm{MHz}$ and emitted a linear polarized near Gaussian-distributed laser beam with a $\mathrm{M}^{2}$ of 1.3 at a central wavelength of $1030 \mathrm{~nm}$. A quarter wave plate was aligned within the laser beam path to obtain circular polarization needed to avoid negative effects in terms of processing quality of the drilled micro holes because of initial linear polarization state $[22,23]$. The pulse duration was measured by means of the auto-correlation method as well as the frequency resolved optical gating method. Both measurement methods provided equivalent results. Figure 1 shows the pulse duration as a function of the PRF. Starting with a pulse duration length of $650 \mathrm{fs}$ at $2 \mathrm{MHz}$, the pulse duration increases with rising PRF and converges at a duration of about $1.2 \mathrm{ps}$ at $48.72 \mathrm{MHz}$. An explanation for this can be found in the non-linear Kerr effect, which lead to a broadening of the spectral bandwidth at higher pulse energies attained at lower PRFs, resulting in a decrease of the pulse duration.

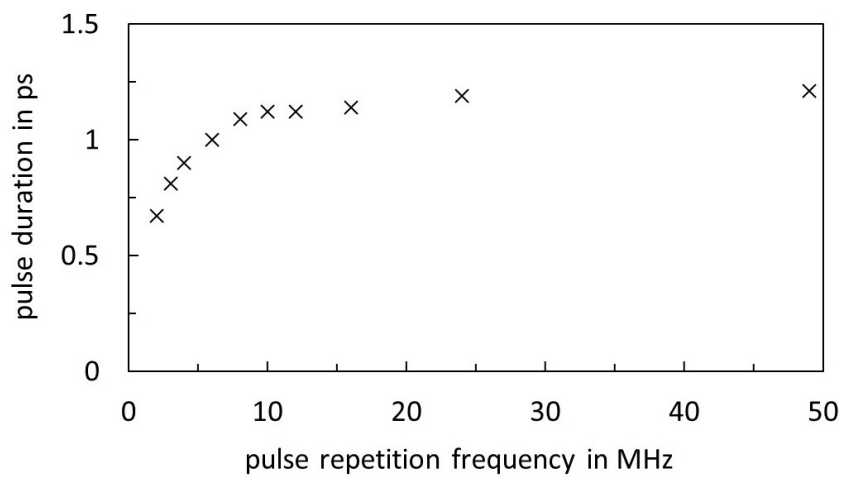

Fig. 1 Pulse duration as a function of the pulse repetition frequency.

For fast lateral beam deflection across the sample surface a galvanometer scan system was utilized. The laser beam was focused onto the material surface by using an f-theta objective with a focal length of $167 \mathrm{~mm}$. The focal spot diameter amounted to $30.5 \mu \mathrm{m}$, that was twice the beam radius $\mathrm{w}_{0}$, corresponding to a Rayleigh length of $0.5 \mathrm{~mm}$. The peak fluence in the experiments was
$\mathrm{H}_{\text {peak }}=12.3 \mathrm{~J} / \mathrm{cm}^{2}$ at a PRF of $\mathrm{f}=1.95 \mathrm{MHz}$, and decreased proportionally with higher PRFs as a consequence of the limited average laser power $\mathrm{P}_{\mathrm{av}}$. The peak fluence was calculated with the following equation:

$$
H_{\text {peak }}=\frac{2 P_{a v}}{\pi w_{0}^{2} f}
$$

All investigations were conducted with the percussion drilling mode and the premise to drill through the material. To determine the number of pulses $n_{p}$ required to drill through a Laser Pulse Controlling system (LPC) was developed and implemented, as shown in the schematic representation in figure 2 . The utilized photodiode was mounted behind the sample substrate. The number of pulses were counted during the drilling process, and after detection of a through hole by the photodiode the laser beam was switched off.

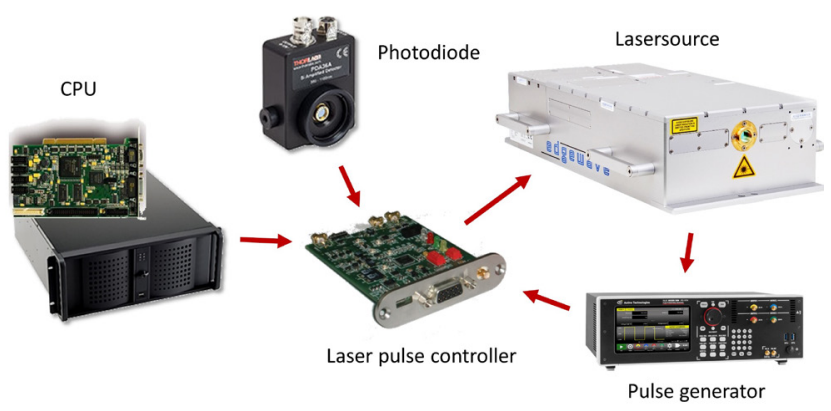

Fig. 2 Scheme of the implemented Laser Pulse Controlling system (LPC).

The sample material used in the investigations was $100 \mu \mathrm{m}$ thick single-side polished, B-doped $<100>$ orientated silicon characterised by a resistivity of $5-10 \Omega \mathrm{cm}$.

The drilled holes were analysed by laser scanning microscopy and scanning electron microscopy (SEM) to evaluate the entrance and exit diameters as well as the micro hole quality. Additionally, cross section views provide information on micro hole formation and micro hole evolution within the material. For statistical analysis, ten holes of each parameter set were produced and analysed. Furthermore, the error bars in the diagrams represent the standard deviation of the mean value of the number of pulses $n_{P}$ necessary to drill through.

\section{Results and discussion}

\subsection{Effects of parameter variation on the number of pulses}

First, the influence of important process parameters on the number of laser pulses necessary to drill through the substrate was studied in detail. Figure 3 depicts the number of irradiated laser pulses needed to drill through as a function of fluence for different PRFs in the range from 1.95 MHz up to 48.72 MHz. For all examined PRFs, it can be seen that the number of pulses needed to drill through the material decreases considerably with an increase of the respective laser fluence, whereby the gradient is getting lower. However, for a frequency of $16.24 \mathrm{MHz}$ at a fluence of $\mathrm{H}_{0}>1 \mathrm{~J} / \mathrm{cm}^{2}$ and a frequency of $9.74 \mathrm{MHz}$ at a fluence of $\mathrm{H}_{0}>2 \mathrm{~J} / \mathrm{cm}^{2}$, an unexpected increase of the number of pulses is visible that has been confirmed by repeating the 
experiment. A potential explanation therefore can be given by plasma and/or particle shielding effects occurring at this specific parameter sets. As a matter of fact, the subsequently irradiated laser pulses will be absorbed and/or scattered by the particle/plasma emissions induced by the previously irradiated pulses thus, in turn, reducing the drilling efficiency. As a general tendency, the statistical variance of the number of pulses needed for drilling through decreases with increasing laser fluence. This can be observed for all investigated PRFs and might be due to the fact that enhanced material removal leads to a higher reproducibility of the drilling process. Another important effect can be seen in figure 3: the silicon sample can be drilled through with significantly lower laser fluence if the PRF was increased. This is a result of heat accumulation, which is more pronounced at higher PRFs due to a limited heat transfer into the surrounding material when subsequent laser pulses irradiate the material in successive shorter time intervals, as also reported in [21, 24-26]. Additionally, in [26] the authors argued that pulse-particle interactions enhance the heat transfer within the drilling hole, which, in turn, reinforces accumulation of heat.

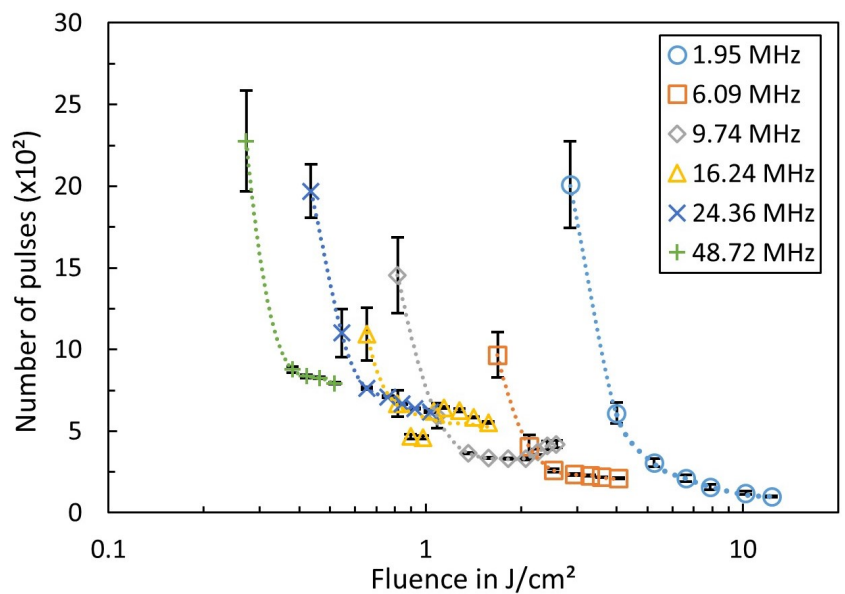

Fig. 3 Required number of pulses to drill through as a function of the fluence for different pulse repetition frequencies.

Table 1 Required number of pulses and related drilling time for through hole generation.

\begin{tabular}{ccccc}
\hline $\begin{array}{c}\text { Frequency } \\
\text { in } \mathrm{MHz}\end{array}$ & $\begin{array}{c}\text { Peak } \\
\text { fluence } \\
\text { in } \mathrm{J} / \mathrm{cm}^{2}\end{array}$ & $\begin{array}{c}\text { Average } \\
\text { power } \\
\text { in W }\end{array}$ & $\begin{array}{c}\text { Required } \\
\text { number } \\
\text { of pulses }\end{array}$ & $\begin{array}{c}\text { Drilling } \\
\text { time } \\
\text { in } \mu \mathrm{s}\end{array}$ \\
\hline 1.95 & 12.32 & 88.3 & 100 & 51.3 \\
6.09 & 4.05 & 90.7 & 212 & 34.8 \\
9.74 & 2.58 & 92.5 & 419 & 43.0 \\
16.24 & 1.55 & 92.6 & 552 & 34.0 \\
24.36 & 1.03 & 92.6 & 618 & 25.4 \\
48.72 & 0.52 & 92.6 & 792 & 16.3
\end{tabular}

Table 1 contains a tabular summary of the number of pulses required to drill through for respective PRFs at maximum available peak fluences. Using a PRF of $1.95 \mathrm{MHz}$ and the equivalent peak fluence of $12.32 \mathrm{~J} / \mathrm{cm}^{2}, 100$ pulses are necessary to drill through the $100 \mu \mathrm{m}$ thick material. In contrast, for a 25 times higher PRF of $48.72 \mathrm{MHz}$ and a corresponding lower peak fluence of $0.52 \mathrm{~J} / \mathrm{cm}^{2}$ the number of pulses increases roughly by a factor of 8 to 792 pulses. However, by applying a fluence of $0.52 \mathrm{~J} / \mathrm{cm}^{2}$ at $1.95 \mathrm{MHz}$ it is not possible to drill through the material as the fluence is below the ablation threshold of about $1.3 \mathrm{~J} / \mathrm{cm}^{2}$ at this PRF as also reported in [7]. This indicates heat accumulation is the most important factor for a decreasing ablation threshold at higher PRFs, as already reported for previous studies [27, 28].

\subsection{Effects of parameter variation on the drilling time}

Another important parameter, the drilling time for through hole generation, characterises the efficiency of the laser drilling process depending on chosen processing parameter sets. Figure 4 illustrates the drilling time as a function of the applied laser fluence and PRF. For a given PRF it can be seen clearly that the drilling time reduces significantly with the higher applied laser fluence. Furthermore, the drilling time is also reduced at higher PRFs.

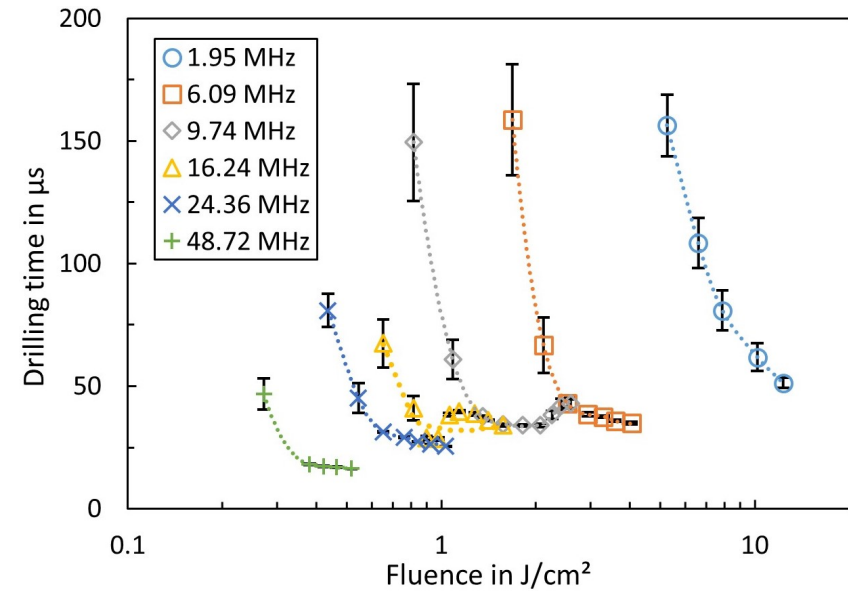

Fig. 4 Drilling time as a function of the fluence for different pulse repetition frequencies.

Table 1 summarises drilling times for through hole generation for the investigated PRF range at respective peak fluences obtained with maximum available average laser power. Generally, the drilling time gradually decreases and, as a consequence of limited maximum average power, the peak fluence lowers if PRF increases. This is a result of heat accumulation effect at PRFs, as discussed in the previous chapter. For example, the drilling time was reduced from $51.3 \mu \mathrm{s}$ to $16.3 \mu \mathrm{s}$ by a factor of 3 for PRFs of $1.95 \mathrm{MHz}$ and $48.72 \mathrm{MHz}$, respectively.

\subsection{Effects of parameter variation on the total energy consumption}

In order to evaluate the efficiency of the drilling process in relation to the energy input, figure 5 depicts the total energy consumption $\mathrm{Q}_{\text {tot }}$ needed for through hole generation, given by

$$
Q_{t o t}=\frac{P_{a v}}{f} n_{p}
$$

which is the accumulated pulse energy reaching the material surface, including the reflected, absorbed and transmitted ratios of the irradiated laser pulses. As can be seen, the energy input decreases by applying higher PRFs as a result of heat accumulation induced by laser-matter-interaction. A closer look at figure 5 reveals a specific fluence for every $\mathrm{PRF}$, which represents a minimum total energy consump- 
tion and hence, the drilling efficiency is optimal, listed in detail in table 2. In comparison, the total energy consumption of $1.23 \mathrm{~mJ}$ at a PRF of $48.72 \mathrm{MHz}$ is 3.7 times lower than $4.52 \mathrm{~mJ}$ at a PRF of $1.95 \mathrm{MHz}$, if the respective optimal laser fluence was applied.

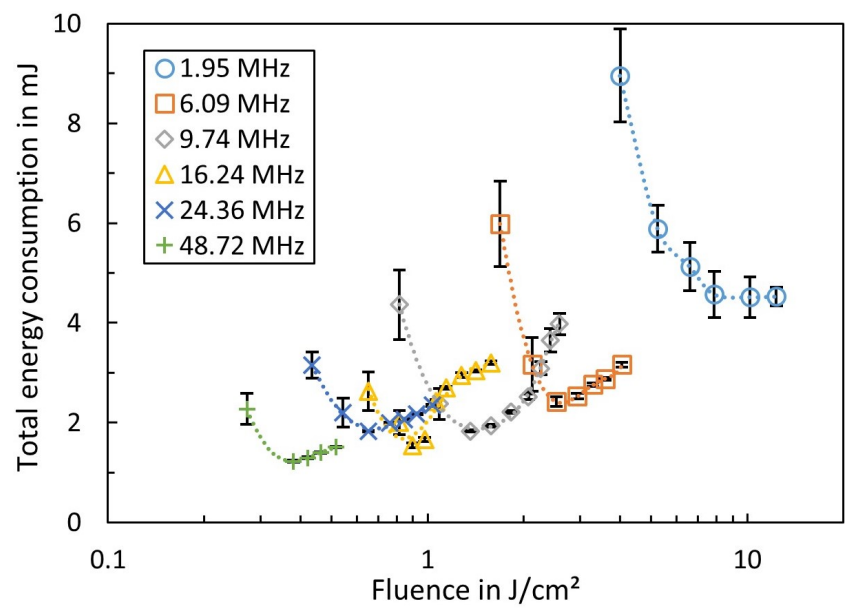

Fig. 5 Total energy consumption as a function of the fluence for different pulse repetition frequencies.

Table 2 Minimum total energy consumption for through hole generation.

\begin{tabular}{ccc}
\hline $\begin{array}{c}\text { Frequency } \\
\text { in MHz }\end{array}$ & $\begin{array}{c}\text { Min. energy con- } \\
\text { sumption in } \mathrm{mJ}\end{array}$ & $\begin{array}{c}\text { equivalent fluence } \\
\text { in } \mathrm{J} / \mathrm{cm}^{2}\end{array}$ \\
\hline 1.95 & 4.52 & 10.2 \\
6.09 & 2.42 & 2.53 \\
9.74 & 1.83 & 1.36 \\
16.24 & 1.54 & 0.90 \\
24.36 & 1.83 & 0.65 \\
48.72 & 1.23 & 0.42 \\
\hline
\end{tabular}

\subsection{Effects of parameter variation onto the hole en- trance and exit diameter}

The diagram in figure 6 illustrates the hole diameter of the entrance and exit side as function of applied laser fluences and PRFs. Generally, it can be seen clearly that the diameters of the hole exit side are significantly smaller than those at the entrance side. This was independently from the chosen PRF, indicating a conical shape of the micro hole cross section. This might be a result of growing plasma shielding, particle shielding and reflection of laser radiation at the hole sidewalls during the drilling process inducing scattering and defocusing the laser beam as reported in [2122]. The micro hole diameter of the entrance side shows a minimum of about $30 \mu \mathrm{m}$, obtained with a laser fluence of $0.4 \mathrm{~J} / \mathrm{cm}^{2}$ at $48.72 \mathrm{MHz}$ and a maximum of about $45 \mu \mathrm{m}$ for a laser fluence of $2.6 \mathrm{~J} / \mathrm{cm}^{2}$ and a PRF of $9.74 \mathrm{~J} / \mathrm{cm}^{2}$. A closer look to the entrance diameters, measured for a given PRF excepting those for $1.95 \mathrm{MHz}$, reveals that the hole diameter depends on the applied laser fluence. Therefore, a minimum hole diameter was obtained for a certain PRF dependent laser fluence. If the given fluence is below this value, the diameter is bigger. This resulted from the higher number of irradiated pulses and, as a consequence, the higher amount of optical energy irradiated to drill through the material. Thus, increased heat accumulation and pronounced melt formation occurs mainly at the micro hole entrance, whereby the melt was probably ejected by the plasma plume as an explanation for bigger entrance diameters. A similar, but less pronounced behaviour could be observed for higher laser fluences, whereby the higher pulse energy is the main reason for increased heat accumulation. It is noteworthy here that the curves for the entrance diameter given in figure 6 show identical tendencies compared with the curves for the total energy consumption presented in figure 5 .

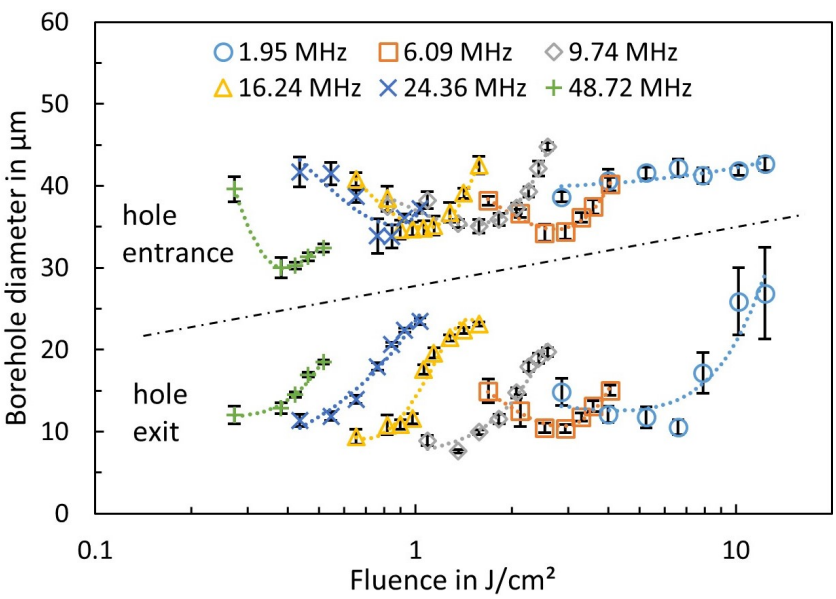

Fig. 6 Micro hole diameter of the hole entrance and exit as a function of the fluence for different pulse repetition frequencies.

The error bars in figure 6 indicate the statistical scattering of the entrance hole diameter as well as the exit hole diameter is very low, especially for high PRFs. Thus, the repeatability of the drilling process is excellent. Furthermore, high-precision micro hole drilling with well-defined entrance and exit side diameters can be performed by choosing appropriate laser parameters. It should be noted, for the lowest applied PRF of $1.95 \mathrm{MHz}$ that the variation of the micro hole exit diameter increases considerably at high fluences. This effect can be explained with the brittleness of silicon: the micro hole exit side bursts a few pulses before drilling through due to laser induced shock waves within the material as a consequence of high energy input at high fluences.

\subsection{Effects of additional pulses on the hole exit diame- ter}

To evaluate the effect of additional pulses irradiated after drilling through, a defined numbers of laser pulses was applied up to a numeric value of 2,000. The results of these investigations are exemplarily shown in figure 7 for the hole exit diameter. The PRF was $48.72 \mathrm{MHz}$. While the entrance diameter was not noteworthy influenced by the number of pulses, the exit diameter gradually increases for a larger number of irradiated laser pulses as expected and converges to a fluence-depending saturation value. This is a result of further material removal after drilling through induced by the outer parts of the Gaussian distributed laser beam. The radial ablation process continues until the outer fluence values fall below the ablation threshold. At this point the hole diameters reach their maximum radial dimension. For example, a maximum exit diameter of $22 \mu \mathrm{m}$ instead of $18.5 \mu \mathrm{m}$ can be achieved by applying a number of 1,600-1,900 laser pulses for a given fluence of $0.52 \mathrm{~J} / \mathrm{cm}^{2}$. 


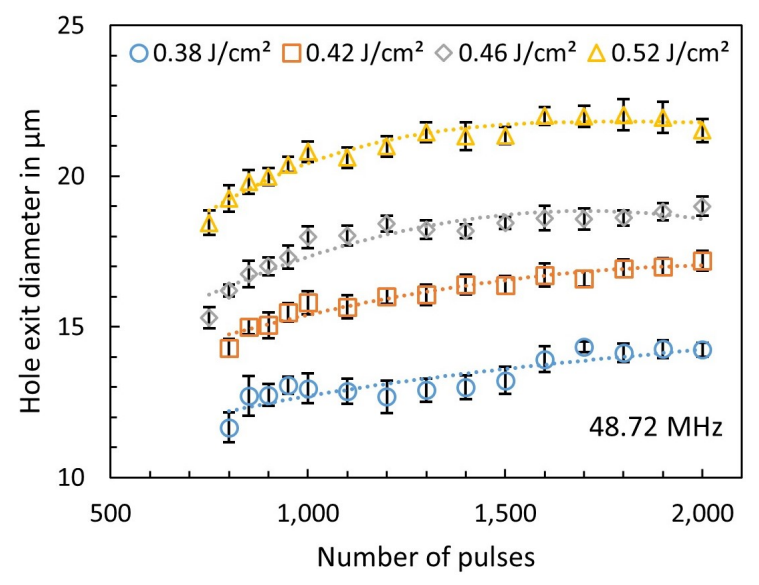

Fig. 7 Hole exit diameter as a function of the defined number of irradiated pulses for different fluences.

Thereby, the exit diameter of the holes can be controlled by the variation of the additional irradiated number of laser pulses. However, extended drilling times and a larger total energy input results in an increased thermal load associated with stronger melting that detrimentally affects this drilling strategy.

\subsection{Effects of parameter variation on the hole quality}

Generally, the drilling quality could be significantly increased by applying laser pulses of higher PRF up to 48.72 MHz. Figure 8 depicts SEM micrographs of the hole entrance and exit side processed with most suitable fluence for high quality in terms of melting residues at the entrance side and the exit side. Note, the micro holes presented in figure 8 are analysed "as processed" without any further post treatment. Using $1.95 \mathrm{MHz}$, the hole entrance is surrounded by a considerable amount of melt and burr. As aforementioned, the exit side shows a chipped hole sidewall in combination with melting residues leading to an undefined hole exit geometry. The associated taper angle was measured to be approximately $14^{\circ}$. At a PRF of 9.74 MHz, spalling could not be observed at the exit side anymore. But the amount of melting residues grows at both sides, the hole entrance and the hole exit. Unexpectedly, the hole quality increases considerably by choosing PRFs of 24.36 MHz and above. Especially for an applied PRF of $48.72 \mathrm{MHz}$, the amount of resolidified melt is negligibly small. There exists only a minimum melt bulge enclosing the hole entrance whereas the hole exit side is completely free of melt. The hole sidewall shows a very smooth structure with sharp and well defined edges. In comparison to lower PRF, the taper angle decreases to a value of about $8^{\circ}$.

As reported in previous studies, laser micro hole drilling of silicon with PRFs up to $26 \mathrm{MHz}$ [7] and in stainless steel up to $10 \mathrm{MHz}$ [26], micro hole quality suffers at those high PRFs as a result of heat accumulation and finally local melting. This is in accordance with the results obtained in this study for PRFs up to $9.74 \mathrm{MHz}$.

Due to the low fluence of $0.52 \mathrm{~J} / \mathrm{cm}^{2}$ applied at a PRF of $48.72 \mathrm{MHz}$ and the resulting short drilling time of $16.3 \mu \mathrm{s}$, the volume affected by heat accumulation is very low in this study. After drilling through the melted and ablated material will be completely ejected out of the hole exit without remaining melting residues.

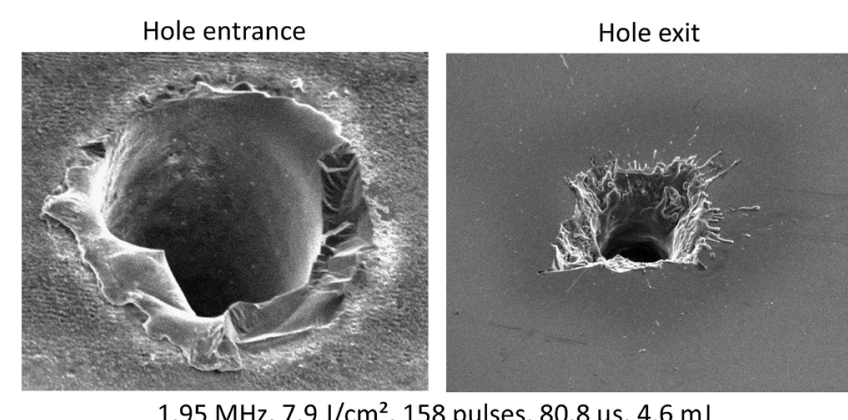

$1.95 \mathrm{MHz}, 7.9 \mathrm{~J} / \mathrm{cm}^{2}, 158$ pulses, $80.8 \mu \mathrm{s}, 4.6 \mathrm{~mJ}$

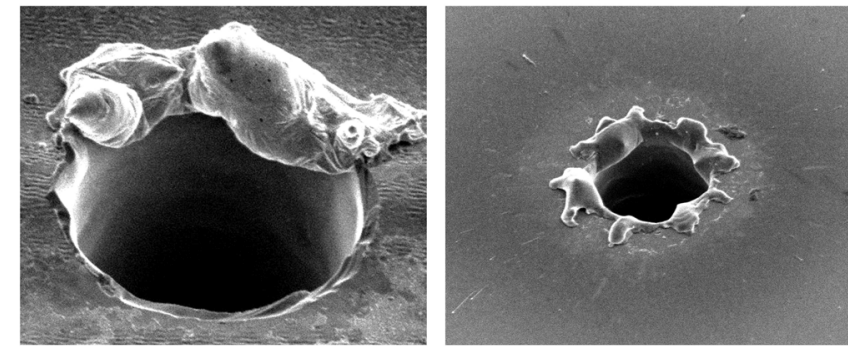

$9.74 \mathrm{MHz}, 2.42 \mathrm{~J} / \mathrm{cm}^{2}, 410$ pulses, $42.1 \mu \mathrm{s}, 3.65 \mathrm{~mJ}$

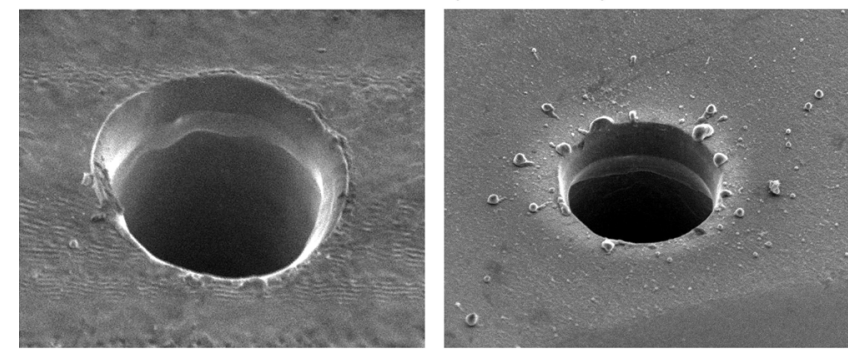

$24.36 \mathrm{MHz}, 1.03 \mathrm{~J} / \mathrm{cm}^{2}, 618$ pulses, $25.4 \mu \mathrm{s}, 2.35 \mathrm{~mJ}$

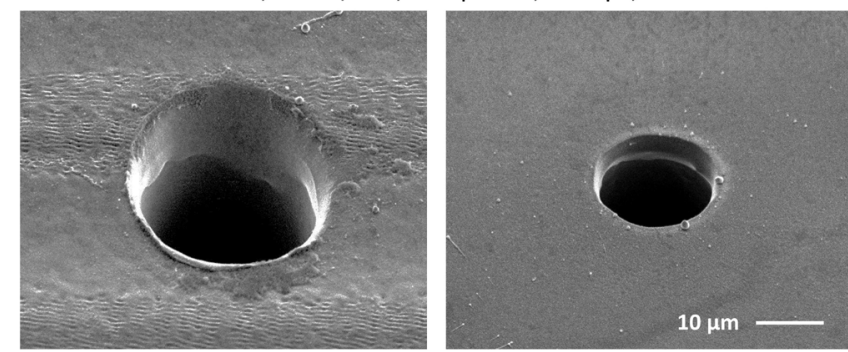

$48.72 \mathrm{MHz}, 0.52 \mathrm{~J} / \mathrm{cm}^{2}, 792$ pulses, $16.3 \mu \mathrm{s}, 1.5 \mathrm{~mJ}$

Fig. 8 SEM images showing the hole entrance and hole exit for various pulse repetition frequencies and respective appropriate parameter settings (from left to right: pulse repetition frequency, fluence, pulse number, drilling time, energy input), SEM tilt angle $45^{\circ}$.

Figure 9 shows SEM micrographs of the micro hole evolution in cross section view using a parameter set for high quality micro hole geometry. To obtain centered crosssectional profiles the samples were cracked at a laser processed predetermined breaking groove along the crystal orientation after micro hole generation. Therefore, the hole entrance side shows an ablation affected area with a depth of about $10 \mu \mathrm{m}$.

As expected, hole formation continues as the number of pulses increases. After a number of 700 pulses a very small hole exit diameter was formed, which was not detected by the LPC. The LPC registered a signal after about 800 pulses comparable to the results with PRF of $48.72 \mathrm{MHz}$ and similar laser fluence in figure 8. It is remarkable, that there is a little constriction near the hole exit, which cannot be explained so far. Moreover, the hole sidewalls appear 
slightly rough during the drilling process. After drilling through the walls seem "smoothed" probably due to the effect of additional pulses after drilling through, which eject out melting residues and ablated particles. Additionally, it can be assumed, that a thin melt film is covering the wall of the hole further contributes to a smoother hole surface.

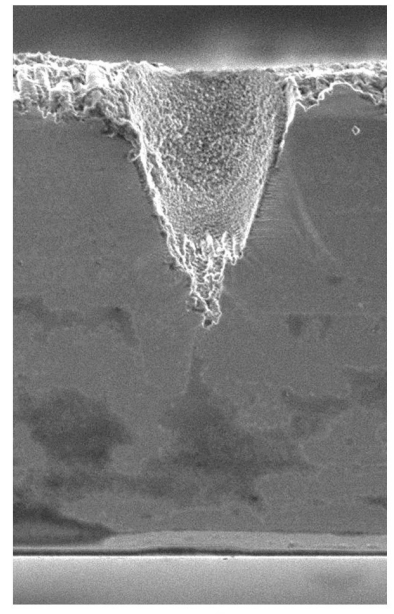

300 pulses

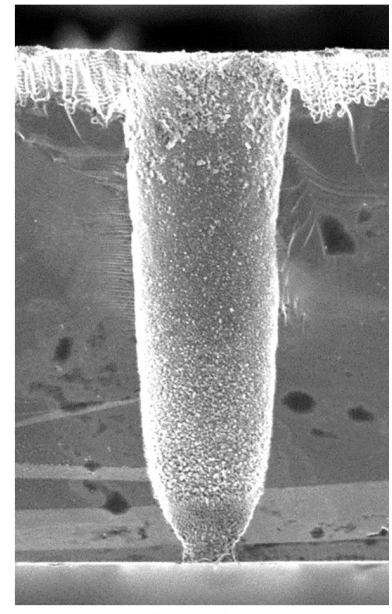

700 pulses

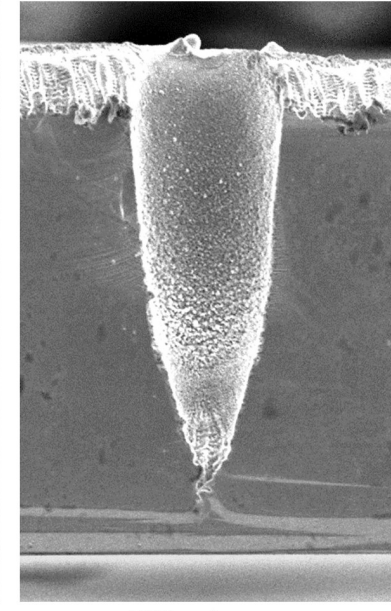

500 pulses

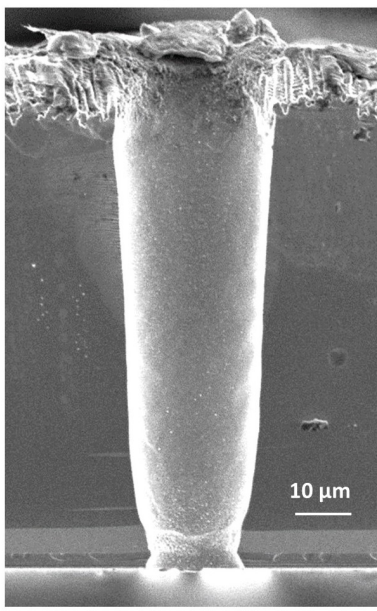

900 pulses
Fig. 9 SEM images showing the cross section micrographs for an increasing number of pulses and a parameter set of $\mathrm{H}_{0}=0.46 \mathrm{~J} / \mathrm{cm}^{2}$ and $\mathrm{f}=48.72 \mathrm{MHz}$

As reported in [21], high PRF laser drilling of stainless steel foils with thicknesses larger than $50 \mu \mathrm{m}$ led to reclosed micro holes due to pronounced melting. Keeping this in mind, on silicon drilling through the material was feasible up to a sample thickness of $100 \mu \mathrm{m}$. As an example, figure 10 illustrates the effect of strong melting and reclosure of the drilled hole for a material thickness of $200 \mu \mathrm{m}$, PRF of $48.72 \mathrm{MHz}$, fluence of $0.46 \mathrm{~J} / \mathrm{cm}^{2}$, and a pulse number of 1,400 . The main reason for this behaviour can be found in the higher number of pulses needed to drill through and therefore an enlarged melting area around the hole entrance as a result of strong heat accumulation. The melt, in turn, flows into the hole supported by plasma pressure, solidifies and recloses the hole near the entrance side. This effect can also be observed for a sample thickness of $100 \mu \mathrm{m}$ at a PRF of $9.74 \mathrm{MHz}$ or higher and applied fluences smaller than the equivalent fluence for minimum energy consumption leading to an increased number of pulses detected to drill through, which is conducive to the heat accumulation effect.
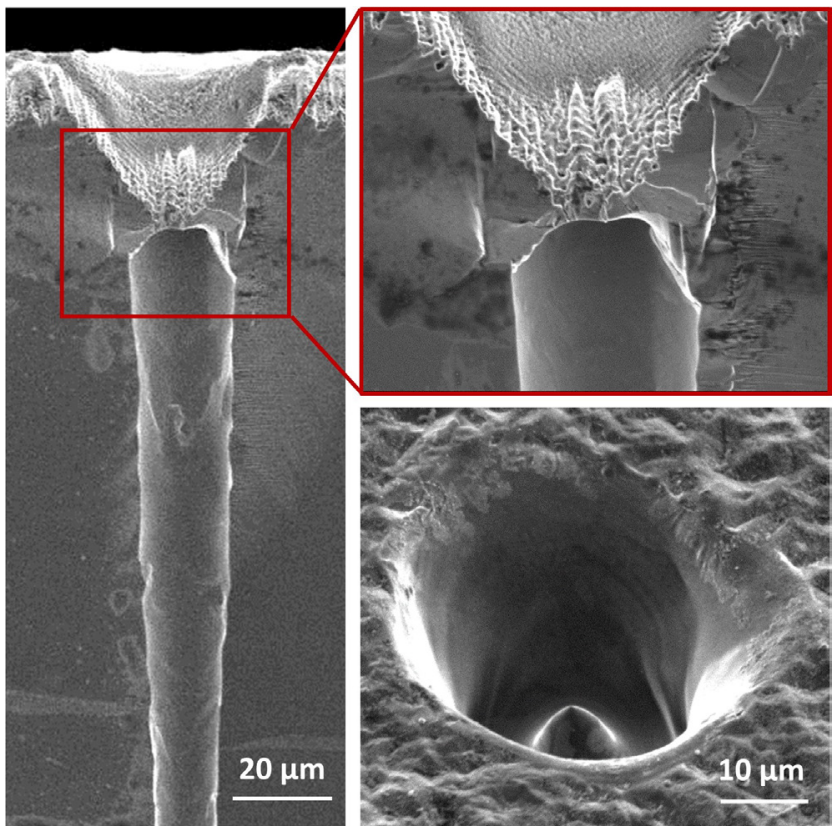

Fig. 10 SEM images with cross section micrographs; material thickness: $200 \mu \mathrm{m}$; number of pulses: 1,400; $\mathrm{H}_{0}=0.46 \mathrm{~J} / \mathrm{cm}^{2}$; $\mathrm{f}=48.72 \mathrm{MHz}$; SEM tilt angle $45^{\circ}$.

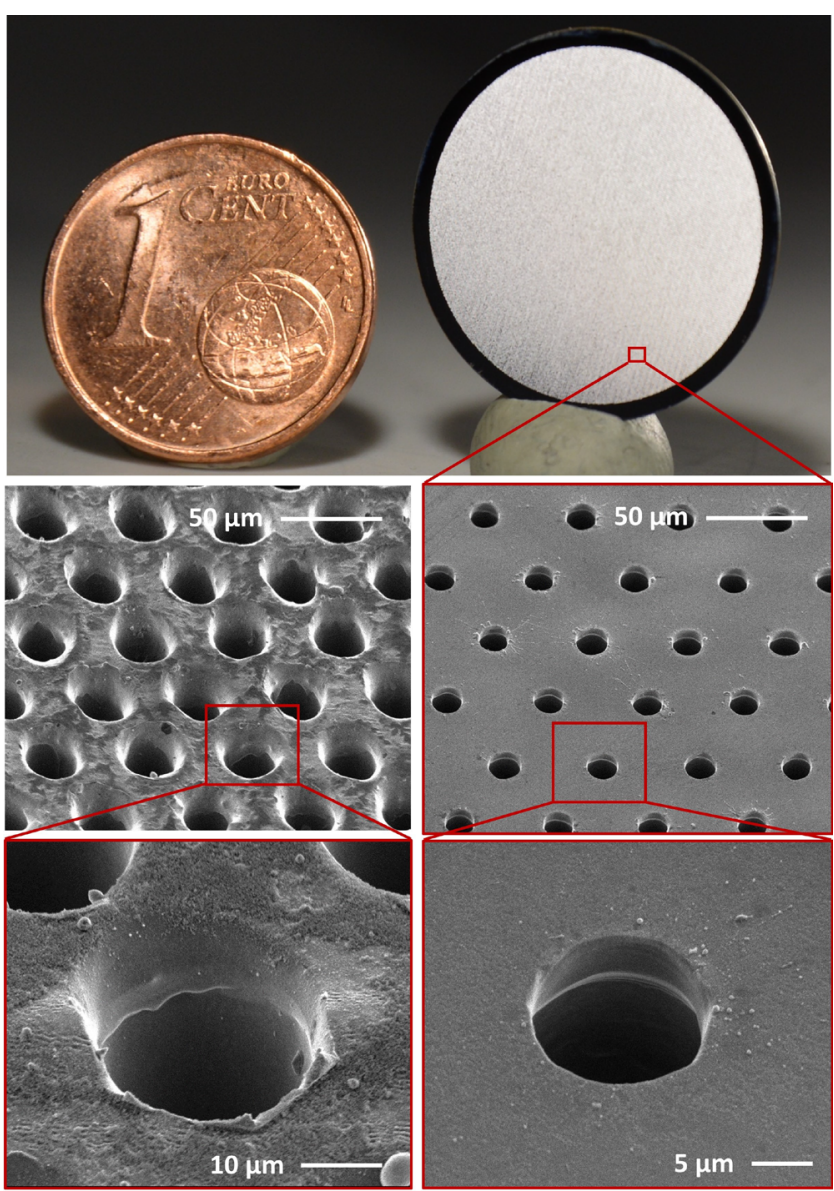

(a)

(b)

Fig. 11 Backlight image and SEM images showing a micro hole grid: $\mathrm{H}_{0}=0.52 \mathrm{~J} / \mathrm{cm}^{2} ; \mathrm{f}=48.72 \mathrm{MHz}$; hole distance: $50 \mu \mathrm{m}$; number of pulses/hole: 850 ; drilling rate: 1000 holes/s; drilled structure diameter: $17 \mathrm{~mm}$; hole number: 104,838; (a) hole entrance; (b) hole exit; SEM tilt angle $45^{\circ}$. 
To prove the feasibility of the high PRF drilling process for industrial applications a hexagonal arranged micro hole grid was generated with optimal parameter sets. The upper image of figure 11 depicts a backlight photography to demonstrate the transparency of the processed hole grid. The holes were processed in a $100 \mu \mathrm{m}$ thick silicon substrate using a fluence of $0.52 \mathrm{~J} / \mathrm{cm}^{2}$ and a PRF of 48.72 MHz. Based on the exit side diameter the drilled area percentage amounts to $14.5 \%$. The demonstrator was analysed "as processed" and only cleaned in an ultrasonic bath after drilling. The grid consists of about 105,000 holes within an outer diameter of $17 \mathrm{~mm}$ corresponding to a spatial hole distance of $50 \mu \mathrm{m}$. For each single hole a number of 850 pulses was applied resulting in a drilling time of $17.4 \mu$ s per hole. Including all delays for beam movement and positioning, the machining time for the presented demonstrator was approximately $105 \mathrm{~s}$. This corresponds to a drilling rate of about 1,000 holes per second. Figure 11 shows also SEM-images providing a closer view onto the drilled grid. As can be seen, only little melting residues cover the hole entrance side whereas the exit side is almost melt free with sharp edges and only little melt splashes in sub- $\mu \mathrm{m}$ dimension. Additionally, the structure is characterised by a high homogeneity of the drilled holes with only little deviations in micro hole geometry and quality.

\section{Conclusion and outlook}

Ultrashort pulse laser sources emitting pulses of high PRF and high average power are an efficient tool to drill precise micro holes, especially micro hole grids of excellent quality. Best results regarding efficiency and quality were obtained by applying laser pulses with the highest available PRF of $48.72 \mathrm{MHz}$ at a fluence of $0.52 \mathrm{~J} / \mathrm{cm}^{2}$ at respective $92 \mathrm{~W}$ average laser power. At this high PRFs, heat accumulation has been identified as a major process influencing effect leading to a more efficient material removal and thus, to lowest drilling times and a minimum total energy consumption in comparison to lower PRFs. In addition, the amount of melt surrounding the micro hole entrance side and exit side is at a minimum for this parameter set.

The micro hole diameter can be modified within limits by varying the fluence, the PRF, or by applying additional pulses after drilling through but at the expense of the micro hole quality. Depending on chosen parameter sets the micro hole diameters are in a range of $30-40 \mu \mathrm{m}$ at the hole entrance side and $10-25 \mu \mathrm{m}$ at the hole exit side. The micro hole cross section is characterised by a conical shape and a smooth surface of the micro hole sidewall. As a disadvantage, the drilling process in silicon is limited to a material thickness of approximately $100 \mu \mathrm{m}$. At higher thickness, a gradual increase of reclosed holes with molten material was observed, intensified with higher PRF.

Micro hole grids of more than 105,000 holes resulting in an effective drilling rate of 1000 holes per second can be generated to demonstrate the feasibility of the high PRF micro drilling process for micro-fabrication and especially, for MEMS GFIS-chip processing. However, the standard thickness of silicon wafers for microsystems fabrication is in the range of $200 \mu \mathrm{m}$ to $500 \mu \mathrm{m}$, which support the longtime stability of the generated highly perforated grids. This is, in turn, a challenging task for future investigation on micro hole grid generation. But the ongoing rapid development on ultrashort pulse laser systems providing high average power at kilowatt level with even higher PRF let expect excellent opportunities to enlarge the parameter window, increase the process efficiency, and probably shift process boundaries to bring the technology to the industry.

\section{Acknowledgement}

The authors thank the European Social Fund for Germany (ESF) for funding project UltraLas (No. 1521030589332).

\section{References}

[1] S. Lee, D. Yang and S. Nikumb: Appl. Surface Science 254, (2008) 2996.

[2] H. Pantsar, H. Herfurth, S. Heinemann, P. Laakso, R. Penttila, Y. Liu and G. Newaz: ICALEO 2008, M507, (2008) 278.

[3] B.N. Chivkov, C. Momma, S. Nolte, F. von Alvensleben and A. Tuennermann: Appl. Phys. A 63, (1996) 109.

[4] P. Laakso, R. Penttilae and P. Heimala: JLMN-Journal of Laser Micro/Nanoengineering Vol. 5, No. 3, (2010) 273.

[5] S. Doering, T. Ullsperger, F. Heisler, S. Richter, A. Tünnermann and S. Nolte: Physics Procedia 41, (2013) 431.

[6] B. Tan: J. Micromech. Microeng. 16, (2006) 109.

[7] B. Tan, S. Panchatsharam and K. Venkatakrishnan: J. Phys. D: Appl. Phys. 42, (2009) 065102.

[8] B. Tan and K. Venkatakrishnan: Journal of Vacuum Science \& Technology B 24, (2006), 211.

[9] D. Shin, J. Suh and Y. Cho: JLMN-Journal of Laser Micro/Nanoengineering Vol. 7, No. 2, (2012) 137.

[10]M. Tajmar and S. Staemm: Proceedings of the $4^{\text {th }}$ Spacecraft Propulsion Conference, (2014) 2967499.

[11] T.C. Hicks: Master's thesis, Naval postgraduate school, Monterey, California, (2008)

[12] L.F. Velasquez-Garcia and A.I. Akinwande: IEEE $21^{\text {st }}$ International Conference on MEMS, (2008) 742.

[13]L.F. Velasquez-Garcia, B.L.P. Gassend and A.I. Akinwande: Journal of Microelectromechanical Systems Vol. 19, Issue 3, (2010) 484.

[14]D. Bock, M. Ebert, F. Roesler, M. Koessling and M. Tajmar: Proceedings of the $5^{\text {th }}$ Russian-German Conference on Electric Propulsion, (2014)

[15]D. Bock, C. Drobny, P. Laufer, M. Koessling and M. Tajmar: 52 ${ }^{\text {nd }}$ AIAA Joint Propulsion Conference, AIAA-2016-4848, (2016)

[16] M. Tajmar P. Laufer and D. Bock: J. Phys.: Conf. Series 840, (2017) 012012.

[17]A. Gruner: Project thesis, TU Dresden, ILR-RSN GB 12-14 (2013)

[18] A. Ancona, F. Roeser, K. Rademaker, J. Limpert, S. Nolte and A. Tuennermann: Opt. Express Vol. 16, No. 12, (2008) 8958.

[19] S. Doering, A. Ancona, S. Haedrich, J. Limpert, S. Nolte and A. Tuennermann: Appl. Phys. A 100, (2010) 53.

[20]A. Ancona, S. Doering, C. Jauregi, F. Roeser, J. Limpert, S. Nolte and A. Tuennermann: Opt. Letters Vol. 34, No. 21, (2009) 3304. 
[21] A. Gruner, J. Schille and U. Loeschner: Proceedings of SPIE 10520, (2018)

[22]A. Gruner, J. Schille and J. Loeschner: Physics Procedia 83, (2016) 157.

[23] K. Venkatakrishnan, B. Tan, P. Stanley and N.R. Sivakumar: J. of Appl. Phys. 92, (2002) 1604.

[24] D.J. Foerster, R. Weber and T. Graf: Proceedings of LPM2017, (2017)
[25]R. Weber, T. Graf, P. Berger, V. Onuseit, M. Wiedenmann, C. Freitag and A. Feuer: Opt. Express Vol. 22, (2014) 11312.

[26] J. Finger and M. Reininghaus: Opt. Express Vol. 22, No. 15, (2014) 18790.

[27] E.G. Gamaly, A.V. Rode, B. Luther-Davies and V.T. Tikhonchuk: Phys. Plasmas 9, (2002) 949.

[28] X.C. Wang, G.C. Lim, H.Y. Zheng, F.L. Ng, W. Liu and S.J. Chua: Appl. Surf. Sci. 228, (2004) 221.

(Received: June 14, 2019, Accepted: September 29, 2019) 